\title{
Safety of celecoxib versus traditional nonsteroidal anti-inflammatory drugs in older patients with arthritis
}

This article was published in the following Dove Press journal: Journal of Pain Research

\author{
Sooyoung Shin ${ }^{1,2}$ \\ 'Department of Clinical Pharmacy, \\ College of Pharmacy, Ajou University, \\ Yeongtong-gu, Suwon, Republic \\ of Korea; ${ }^{2}$ Research Institute \\ of Pharmaceutical Science and \\ Technology (RIPST), Ajou University, \\ Yeongtong-gu, Suwon, Republic of \\ Korea
}

Background: A 2011 systematic review found an increased cardiovascular (CV) risk at both $\leq 200 \mathrm{mg}$ /day and $>200 \mathrm{mg} /$ day doses of celecoxib. This study aimed to evaluate adverse drug events with celecoxib relative to traditional nonsteroidal anti-inflammatory drugs (NSAIDs) in real-world practice settings, focusing on gastrointestinal (GI), CV, and renal toxicity, in older patients with osteoarthritis or rheumatoid arthritis.

Methods: In this population-based retrospective cohort study using national health insurance claims data in Korea, patients aged 65 years and older with arthritis who were treated with celecoxib or traditional NSAIDs for $\geq 30$ days in 2016, were included for study analyses. The primary outcome was hospital encounter for GI bleeding associated with celecoxib vs traditional NSAIDs use. The secondary outcomes included a composite of CV diseases, coronary revascularization, and incident renal events.

Results: After 1:1 propensity score matching, 73,748 patients in each cohort were identified for study entry. Celecoxib treatment which lasted for $\geq 120$ days was associated with a lower risk of GI bleeding than traditional NSAIDs $(\mathrm{OR}=0.84, P=0.03)$. Such a relationship was not observed in shorter treatment strata and overall in all strata combined. When patients with gastroprotective prophylaxis were excluded from subgroup analysis, no evidence of improved GI tolerability was observed with celecoxib. $\mathrm{CV}$ and renal risks appeared higher with celecoxib than with traditional NSAIDs ( $\mathrm{OR}=1.08, P<0.001$ and $\mathrm{OR}=1.22, P<0.001$, respectively). About $4.7 \%$ of celecoxib users received a higher than maximum dose ( $400 \mathrm{mg} /$ day); a dose-dependent increase in $\mathrm{CV}$ and renal risks was assessed with celecoxib.

Conclusion: Celecoxib was associated with decreased risk of GI bleeding compared with traditional NSAIDs when treatment lasted for $\geq 120$ days, but such a relationship was not found among subgroup patients with no concomitant use of gastroprotective prophylaxis. Celecoxib users were more likely to experience CV and renal events than traditional NSAIDs users, and a dose-dependent risk relationship was observed with celecoxib.

Keywords: celecoxib, nonsteroidal anti-inflammatory drug, NSAID, adverse drug events, osteoarthritis, rheumatoid arthritis

\section{Introduction}

Older patients with osteoarthritis or rheumatoid arthritis often require long-term treatment with nonsteroidal anti-inflammatory drugs (NSAIDs) for the relief of chronic pain and inflammation. ${ }^{1,2}$ With their decreased renal function and gastrointestinal (GI) physiological changes progressing with aging, those patients are more predisposed to adverse effects of prolonged exposure to the ulcerogenic analgesics. It is well documented that the GI toxicity of NSAIDs is associated with the nonselective inhibition
Correspondence: Sooyoung Shin of Pharmacy, Ajou University, 206, World cup-ro, Yeongtong-gu, Suwon, Gyeonggi-do 16499, Republic of Korea $\mathrm{Tel}+82312193456$

Fax +82312193435

Email syshin@ajou.ac.kr
Journal of Pain Research 2018:1 I 3211-3219

(c) $(1) \circledast 2018$ Shin. This work is published and licensed by Dove Medical Press Limited. The full terms of this license are available at https://www.dovepress.com/term

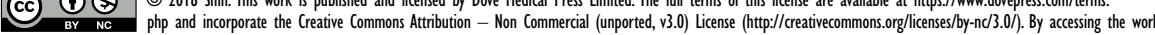
you herbby accept the Terms. Non-commercial uses of the work are permitted without any further permission from Dove Medical Press Limited, provided the work is properly attributed. For permission for commercial use of this work, please see paragraphs 4.2 and 5 of our Terms (https:/www.dovepress.com/terms.php). 
of the two cyclooxygenase (COX) isoforms, where COX-1 inhibition prevents the production of GI protective prostaglandins resulting in higher risk of ulcerogenic adverse events, while COX-2 inhibition exerts anti-inflammatory and analgesic effects. ${ }^{3,4}$ Traditional NSAIDs are mostly a dual inhibitor of both COX isoforms, and hence can cause GI toxicities. Celecoxib, however, by selectively inhibiting COX-2, has the potential to reduce GI adverse effects, but previous studies suggested that the unbalanced inhibition of COX or prostaglandin synthesis may pose a greater risk of cardiovascular (CV) adverse outcomes. ${ }^{5,6}$

Due to the controversies over CV safety of COX-2 specific inhibitors, rofecoxib and valdecoxib were withdrawn from the market in 2004 and 2005, respectively. ${ }^{5}$ The only remaining coxib agent, celecoxib, has been made continuously available for clinical use, despite the 2005 clinical trial results that suggested increased CV risks with its use. ${ }^{7}$ Such a relationship was deemed inconclusive in light of a low incidence of $\mathrm{CV}$ events and higher-than-normal doses used for celecoxib in the trial. The US Food and Drug Administration (FDA), however, required an additional clinical investigation of reassurance regarding celecoxib's CV safety; the 2016 study showed that celecoxib was not inferior to ibuprofen nor to naproxen in terms of CV safety outcomes. ${ }^{8}$ However, although the safety of moderate doses of celecoxib (200-400 mg daily) has been supported by the postmarketing trial findings, whether higher doses, often encountered in real-world clinical practice where regulatory restrictions on its doses are not put in place, would also be safe remains uncertain. ${ }^{9}$

The aim of this study was to evaluate adverse drug events with celecoxib as compared to traditional NSAIDs, focusing on GI, CV, and renal toxicity, in older patients with osteoarthritis or rheumatoid arthritis, using National Health Insurance (NHI) data in Korea.

\section{Methods}

\section{Study population}

A population-based retrospective cohort study involving older adults with osteoarthritis or rheumatoid arthritis was conducted using the Korean Health Insurance Review \& Assessment Service-Aged Patient Sample-2016 (HIRAAPS-2016-0062), which contains NHI claims data on 1,327,455 older patients (approximately $20 \%$ of the older patient population in Korea) with ensured national representativeness. The HIRA database saves administrative patient information on healthcare services offered for the entire national beneficiaries, including patient demographics, diagnoses per the International Classification of Disease, 10th Revision (ICD-10), procedures, and comprehensive medical utilization, along with prescription data. The initial patient samples were selected using a stratified randomized sampling method, and, of those, older patients with osteoarthritis or rheumatoid arthritis were identified if patients 65 years and older had a record of osteoarthritis (ICD-10 code M15.x-19.x) or rheumatoid arthritis (ICD-10 code M05.xM06.x) diagnoses. The study protocol was approved by the Institutional Review Board of Ajou University (201804HB-EX-001). Informed consent from study participants was waived due to the retrospective design of the study based on health insurance data. No further ethics approval was needed because the investigator was authorized by the HIRA to analyze anonymized patient data for research purposes.

\section{Study medications and covariates}

Older patients requiring daily analgesic treatment based on either celecoxib or traditional NSAIDs to control osteoarthritis- or rheumatoid arthritis-induced pain were selected for study inclusion. All eligible prescription data were captured using a total of 35 relevant substance codes of orally administered agents with different strengths and formulations listed in the national formulary. Individual dual COX-1/ COX-2 inhibitors plus diclofenac, a COX-2 inhibitor, were tracked and categorized as traditional NSAIDs as a whole, and switches between traditional NSAIDs were permitted. Any therapy with a duration of below 30 days was regarded as temporary use, hence excluded from further analysis. For the maintenance doses assessment, only those therapies with a duration of at least 30 days were included. The total daily dose (in $\mathrm{mg}$ ) was obtained by multiplying the strength of individual active ingredient by the number of tablets or capsules per day, then by the number of prescribed days for each agent. Prespecified covariates included age, sex, primary arthritis diagnosis (osteoarthritis or rheumatoid arthritis), comorbidities, and other relevant pharmacologic therapies. The following comorbid status was identified per ICD-10 codes: diabetes without complications, diabetes with complications, hypertension, dyslipidemia, and Helicobacter pylori infection. Other medications use for disease control or prevention of adverse outcomes were also assessed, including aspirin, antiplatelet, anticoagulant, statin, disease-modifying antirheumatic drug (DMARD), proton pump inhibitor (PPI), and histamine-2 receptor antagonist (H2RA).

\section{Study outcomes}

The primary outcome was the incidence and risk of GI bleeding in celecoxib-treated patients vs traditional NSAIDs-treated 
patients, stratified by differential treatment duration to account for the cumulative effect of prolonged therapy. The endpoint event was captured via healthcare visit episodes for GI bleeding, and subsequently between-group differences in GI bleeding risk were assessed. To better evaluate the sole effects of celecoxib vs traditional COX inhibitors on GI complications, a subgroup analysis was designed, where only those patients with no upper GI prophylaxis based on PPIs or H2RAs were selected for risk analysis. The secondary $\mathrm{CV}$ disease outcome was a composite of hospital encounter with a primary diagnosis of ischemic stroke, transient ischemic attack, unstable angina, acute myocardial infarction, chronic ischemic heart disease, and heart failure. Hospital visit episodes for coronary revascularization, identified with procedure codes for percutaneous coronary intervention (PCI) with stent implantation and for coronary artery bypass graft (CABG), along with any visit episodes for incident renal events (hypertensive renal disease, nephritic syndrome, chronic kidney disease, kidney failure, renal dialysis, and disorders resulting from impaired renal tubular function), were also captured for additional secondary outcome analysis.

\section{Statistical analysis}

A multivariable logistic regression model incorporating all baseline variables (age category, sex, primary arthritis diagnosis, comorbidity, and medications use history) as covariates was employed to yield a propensity score (PS) value for individual patients, which predicts the probability of patient exposure to celecoxib vs traditional NSAIDs in the setting of the pretreatment characteristics. Study patients were then PS-matched to celecoxib or traditional NSAIDs in a 1:1 ratio to balance baseline covariates (or potential confounders) between the two cohorts. Then, odds ratios (ORs) and 95\% confidence intervals (CIs) for adverse GI, CV, and renal endpoint events associated with study therapy exposure were estimated. The PS-matched patients in the two comparison groups were further stratified by treatment duration into the following three strata: 30-59 days, 60-119 days, and 120 or more days. $P$-values were two-tailed and considered to be statistically significant if less than 0.05. Maintenance daily doses of commonly prescribed study agents were also assessed for descriptive purposes. All statistical analyses were performed using SAS version 9.4 (SAS Institute Inc., Cary, NC).

\section{Results}

\section{Characteristics of study patients}

Of the total number of Korean older patients registered in the HIRA database in 2016 (approximately 6.6 million), the number of patients included in the initial older patient sample was 1,327,455 (female 58.1\%). Of those, patients aged 65 years and above with a history of osteoarthritis or rheumatoid arthritis who received celecoxib- or traditional NSAIDs-based analgesic therapy for at least 30 days were selected, hence resulting in a total of 183,022 patients eligible for study entry. A total of 13,144 patients were then excluded from further analysis as they had received both celecoxib and traditional NSAIDs treatment for an equivalent number of days (treatment duration not differing by more than $50 \%$ of each other's prescribed days). Of the resulting 169,878 patients $(73,748$ in the celecoxib cohort vs 96,130 in the traditional NSAIDs cohort), 1:1 PS matching was performed, leaving 73,748 patients in each comparison group. Baseline attributes of the PS-matched study patients are summarized in Table 1. Female patients outnumbered males by about threefold ( $74.3 \%$ vs $25.7 \%$ ). Overall, the distribution of age category, sex, primary arthritis diagnosis, comorbidity, and other medications use was balanced between the two treatment cohorts following PS-matching. The breakdown of all

Table I Baseline characteristics of older patients with osteoarthritis or rheumatoid arthritis (I:I PS-matched celecoxib and traditional NSAIDs cohorts)

\begin{tabular}{|c|c|c|}
\hline Characteristic & $\begin{array}{l}\text { Celecoxib } \\
(N=73,748)\end{array}$ & $\begin{array}{l}\text { Traditional } \\
\text { NSAIDs } \\
(\mathrm{N}=73,748)\end{array}$ \\
\hline Age, years, mean $\pm S D$ & $75.7 \pm 6.4$ & $75.2 \pm 6.3$ \\
\hline 65 to $<75, \mathrm{n}(\%)$ & $34,473(46.7)$ & $36,236(49.1)$ \\
\hline 75 to $<85, \mathrm{n}(\%)$ & $31,762(43.1)$ & $31,266(42.4)$ \\
\hline$\geq 85, \mathrm{n}(\%)$ & $7,513(10.2)$ & $6,246(8.5)$ \\
\hline Female sex, n (\%) & $57,623(78.1)$ & $51,914(70.4)$ \\
\hline \multicolumn{3}{|l|}{ Primary arthritis diagnosis, $\mathrm{n}(\%)$} \\
\hline Osteoarthritis & $58,794(79.7)$ & $61,324(83.2)$ \\
\hline Rheumatoid arthritis & $3,797(5.2)$ & $3,133(4.2)$ \\
\hline Both arthritis & $11,157(15.1)$ & $9,291(12.6)$ \\
\hline \multicolumn{3}{|l|}{ Comorbidity, n (\%) } \\
\hline Diabetes without complications & $28,785(39.0)$ & $27,57 \mid(37.4)$ \\
\hline Diabetes with complications & $11,702(15.9)$ & $10,536(14.3)$ \\
\hline Hypertension & $54,613(74.1)$ & $53,630(72.7)$ \\
\hline Dyslipidemia & $49,597(67.3)$ & $47,299(64.1)$ \\
\hline Helicobacter pylori infection & $313(0.4)$ & $319(0.4)$ \\
\hline \multicolumn{3}{|l|}{ Other medications, n (\%) } \\
\hline Aspirin & $19,517(26.5)$ & $19,097(25.9)$ \\
\hline Antiplatelet & $14,465(19.6)$ & $14,050(19.1)$ \\
\hline Anticoagulant & $2,113(2.9)$ & $\mathrm{I}, 783(2.4)$ \\
\hline Statin & $31,015(42.1)$ & $29,635(40.2)$ \\
\hline DMARD & $3,594(4.9)$ & $2,040(2.8)$ \\
\hline PPI & $27,225(36.9)$ & $25,616(34.7)$ \\
\hline Histamin-2 receptor antagonist & 37,07 I (50.3) & $39,592(53.7)$ \\
\hline
\end{tabular}

Abbreviations: DMARD, disease-modifying antirheumatic drug; NSAIDs, nonsteroidal anti-inflammatory drugs; PPI, proton pump inhibitor; PS, propensity score. 
comparator NSAIDs collectively categorized as traditional NSAIDs along with celecoxib is summarized per prescription volume and provided in the supplementary material (Table S1).

\section{Gl bleeding outcomes}

The incidence and risk of GI bleeding depending on analgesic treatment were assessed in the overall PS-matched study patients as well as separately according to stratification by treatment duration into the three strata (Table 2). Overall, the primary outcome event was encountered in $781(1.1 \%)$ and $816(1.1 \%)$ patients in the celecoxib and traditional NSAIDs cohorts, respectively; the odds of hospital encounter for GI bleeding were not statistically significantly lower in celecoxib-treated patients than in traditional NSAIDs-treated patients when therapy lasted for 30 days or more ( $\mathrm{OR}=0.96 \%$, $95 \% \mathrm{CI}=0.87-1.06, P=0.38)$. In the last stratum (treatment days of 120 or more), however, celecoxib users were less likely to experience GI bleeding compared with traditional NSAIDs users (OR=0.84\%, 95\% CI $=0.72-0.99, P=0.03$ ).

In the subgroup analysis, those patients who received prophylactic gastroprotection based on PPIs or H2RAs were excluded, leaving 23,850 patients in each treatment groups post PS-matching (Table 3). The risk analysis was repeated in the subgroup patients to better evaluate unconfounded effects of study treatment exposure on GI toxicity risk by eliminating any influence of gastroprotective agents, ${ }^{10,11}$ using analogous stratification methods applied in the prior primary analysis. No OR values in all strata of treatment duration were associated with statistical significance; when treatment duration of 30 days and more was all considered for risk analysis, the overall OR ( $95 \% \mathrm{CI})$ for GI bleeding was 1.27 (0.98-1.65) with a $P$-value of 0.07 , only approaching borderline significance.

\section{$\mathrm{CV}$ and renal outcomes}

Table 4 summarizes the results of incidence rates and comparative risk of secondary endpoints of the prespecified CV and renal events among the PS-matched study patients with therapy duration of at least 120 days; the ORs associated with each component of $\mathrm{CV}$ composite endpoint were also presented. Overall, celecoxib users showed an elevated risk of hospital encounter for $\mathrm{CV}$ diseases as well as renal events compared with traditional NSAID users: the ORs (95\% CIs) were $1.08(1.04-1.12)$ and $1.22(1.11-1.35)$, respectively. The risk of $\mathrm{CV}$ diseases showed only a near-significant trend

Table 2 PS-matched analysis for relative risks of GI bleeding associated with celecoxib vs traditional NSAIDs (stratified into three strata by treatment duration)

\begin{tabular}{|c|c|c|c|c|}
\hline GI bleeding & Celecoxib $(\mathrm{N}=73,748)$ & Traditional NSAIDs $(\mathrm{N}=\mathbf{7 3 , 7 4 8 )}$ & \multicolumn{2}{|c|}{ Celecoxib vs traditional NSAIDs } \\
\hline Therapy duration & \multicolumn{2}{|c|}{ Number of events/number of patients (percent) } & OR (95\% Cls) & $P$-value \\
\hline Overall & $781 / 73,748(1.1)$ & $816 / 73,748(1.1)$ & $0.96(0.87-1.06)$ & 0.38 \\
\hline 30-59 days & $193 / 18,003(1.1)$ & $327 / 32,228(1.0)$ & $1.06(0.88-1.26)$ & 0.54 \\
\hline $60-119$ days & $222 / 19,903(1.1)$ & $233 / 20,39 I(I . I)$ & $0.98(0.8 \mathrm{I}-\mathrm{I} .17)$ & 0.80 \\
\hline $120+$ days & $366 / 35,842(1.0)$ & $256 / 2 I, 129(1.2)$ & $0.84(0.72-0.99)$ & 0.03 \\
\hline
\end{tabular}

Notes: $P$-values were estimated by chi-squared test. A statistically significant $P$-value is highlighted in bold.

Abbreviations: $\mathrm{Cl}$, confidence interval; $\mathrm{Gl}$, gastrointestinal; NSAIDs, nonsteroidal anti-inflammatory drugs; OR, odds ratio; PS, propensity score.

Table 3 PS-matched subgroup analysis for relative risk of GI bleeding associated with celecoxib vs traditional NSAIDs in patients with no concomitant PPI or H2RA use (stratified into three strata by treatment duration)

\begin{tabular}{|c|c|c|c|c|}
\hline GI bleeding & Celecoxib $(\mathrm{N}=\mathbf{2 3 , 8 5 0})$ & Traditional NSAIDs $(\mathbf{N}=\mathbf{2 3 , 8 5 0})$ & \multicolumn{2}{|c|}{ Celecoxib vs traditional NSAIDs } \\
\hline Therapy duration & \multicolumn{2}{|c|}{ Number of events/number of patients (percent) } & OR (95\% Cls) & $P$-value \\
\hline Overall & $127 / 23,850(0.5)$ & $100 / 23,850(0.4)$ & $1.27(0.98-1.65)$ & 0.07 \\
\hline 30-59 days & $31 / 6,329(0.5)$ & $44 / 11,206(0.4)$ & $1.25(0.79-1.98)$ & 0.34 \\
\hline $60-119$ days & $32 / 6,156(0.5)$ & $23 / 6,078(0.4)$ & $1.38(0.80-2.35)$ & 0.24 \\
\hline $120+$ days & $64 / 11,365(0.6)$ & $33 / 6,566(0.5)$ & $\mathrm{I} .12(0.74-I .7 I)$ & 0.59 \\
\hline
\end{tabular}

Notes: $P$-values were estimated by chi-squared test.

Abbreviations: $\mathrm{Cl}$, confidence interval; GI, gastrointestinal; H2RA, histamine-2 receptor antagonist; NSAIDs, nonsteroidal anti-inflammatory drugs; OR, odds ratio; PPI, proton pump inhibitor; PS, propensity score. 
Table 4 PS-matched analysis for relative risks of CV and renal events associated with celecoxib vs traditional NSAIDs (treatment duration for 120 or more days)

\begin{tabular}{|c|c|c|c|c|}
\hline \multirow[t]{2}{*}{ Outcomes } & Celecoxib $(\mathrm{N}=35,842)$ & Traditional NSAIDs $(\mathbf{N}=2 \mathrm{I}, 129)$ & \multicolumn{2}{|c|}{ Celecoxib vs traditional NSAIDs } \\
\hline & \multicolumn{2}{|c|}{ Number of events (percent) } & OR $(95 \% \mathrm{Cl})$ & $P$-value \\
\hline CV diseases & $14,379(40.1)$ & 8,101 (38.3) & $1.08(1.04-1.12)$ & $<0.001$ \\
\hline Ischemic stroke & $7,761(21.7)$ & $4,446(21.0)$ & $1.04(0.99-1.08)$ & 0.09 \\
\hline Transient ischemic attack & $1,059(3.0)$ & $660(3.1)$ & $0.94(0.86-1.04)$ & 0.25 \\
\hline Unstable angina & $6,394(17.8)$ & $3,487(16.5)$ & $1.10(1.05-1.15)$ & $<0.001$ \\
\hline Acute myocardial infarction & $721(2.0)$ & $44 I(2.1)$ & $0.96(0.85-1.09)$ & 0.54 \\
\hline Chronic ischemic heart disease & $\mathrm{I}, 85 \mathrm{I}(5.2)$ & $997(4.7)$ & $1.10(1.02-1.19)$ & 0.02 \\
\hline Heart failure & $3,402(9.5)$ & $1,856(8.8)$ & $1.09(1.03-1.16)$ & 0.005 \\
\hline Coronary revascularization & $179(0.5)$ & $118(0.6)$ & $0.89(0.7 I-1.13)$ & 0.34 \\
\hline $\mathrm{PCl}$ & $173(0.5)$ & $117(0.6)$ & $0.87(0.69-1.10)$ & 0.25 \\
\hline CABG & $6(0.02)$ & $2(0.01)$ & NA & NA \\
\hline Renal events & $1,210(3.4)$ & $587(2.8)$ & $1.22(1.11-1.35)$ & $<0.001$ \\
\hline
\end{tabular}

Notes: $P$-values were estimated by chi-squared test. Statistically significant $P$-values are highlighted in bold.

Abbreviations: CABG, coronary artery bypass graft; Cl, confidence interval; CV, cardiovascular; NA, not applicable; NSAIDs, nonsteroidal anti-inflammatory drugs; OR, odds ratio; $\mathrm{PCl}$, percutaneous coronary intervention; PS, propensity score.

when therapy duration of at least 30 days (or all three strata) was assessed collectively for risk analysis $(\mathrm{OR}=1.02 \%, 95 \%$ $\mathrm{CI}=1.00-1.04, P=0.07)$. However, in the stratum of 120 days and more of treatment, statistical significance was reached ( $\mathrm{OR}=1.08 \%, 95 \% \mathrm{CI}=1.04-1.12, P<0.001)$, suggesting more negative $\mathrm{CV}$ effects of celecoxib vs traditional NSAIDs as therapy persisted longer; when individual components of $\mathrm{CV}$ disease endpoint were assessed separately, the odds of hospital encounter due to unstable angina, chronic ischemic heart disease, and heart failure were higher in celecoxibreceiving patients than in traditional NSAIDs-receiving patients: the ORs (95\% CIs) were 1.10 (1.05-1.15), 0.10 (1.02-1.19), and 1.09 (1.03-1.16), respectively. However, the risk of hospitalization for coronary revascularization was not significantly different between the two cohorts $(P=0.34)$. Due to the low rates of $\mathrm{CABG}$, albeit numerically more frequent in the celecoxib group than in the traditional NSAIDs group, its OR results are not presented in Table 4.

\section{Maintenance dose of celecoxib and traditional NSAIDs}

The maintenance doses of celecoxib and commonly used oral traditional NSAID agents (loxoprofen, dexibuprofen, ibuprofen, and naproxen) were assessed using descriptive statistics and are visually depicted in Figure 1. Here, all relevant prescriptions with therapy duration of at least 30 days were included for assessment. The interquartile range of maintenance dosage of the five agents appeared to fall within the upper limits of the recommended daily dose for their anti-inflammatory properties. Of all the COX inhibitors included in this study, the most commonly-used agent per prescription volume was loxoprofen, followed by celecoxib (31.6\% and $12.4 \%$, respectively, as shown in Table S1): the median dose (interquartile range) was $180 \mathrm{mg} /$ day (180-180 $\mathrm{mg} /$ day) and $200 \mathrm{mg} /$ day (200-400 mg/day), respectively. About $4.7 \%(350 / 73,748)$ of patients on celecoxib treatment received a total daily dose of greater than $400 \mathrm{mg}$ (the maximum daily dose); of those, $71.4 \%$ (250/350) of patients had osteoarthritis, with no comorbidity history of rheumatoid arthritis. Among the osteoarthritis-only patients on celecoxib, about $24.3 \%(14,309 / 58,794)$ patients were prescribed with a daily dose of more than $200 \mathrm{mg} /$ day (the maximum daily dose recommended for osteoarthritis patients).

In an additional analysis on comparative risk associated with high-dose (more than $400 \mathrm{mg} /$ day) vs low-to-moderatedose (less than or equal to $400 \mathrm{mg} /$ day) celecoxib among those patients on celecoxib for at least 30 days, high-dose receiving patients were more likely to encounter GI bleeding, a composite endpoint of $\mathrm{CV}$ diseases, and renal events relative to those receiving a low-to-moderate dose of celecoxib: the ORs (95\% CIs) were 2.77 (1.47-5.22), 1.44 (1.17-1.78), and 1.97 (1.28-3.05), respectively (Table 5).

\section{Discussion}

In this study, celecoxib treatment lasting for 120 days or more was associated with a lower risk of GI bleeding than traditional NSAIDs $(\mathrm{OR}=0.84, P=0.03)$, in accordance with the potential GI advantages attributable to the specific 


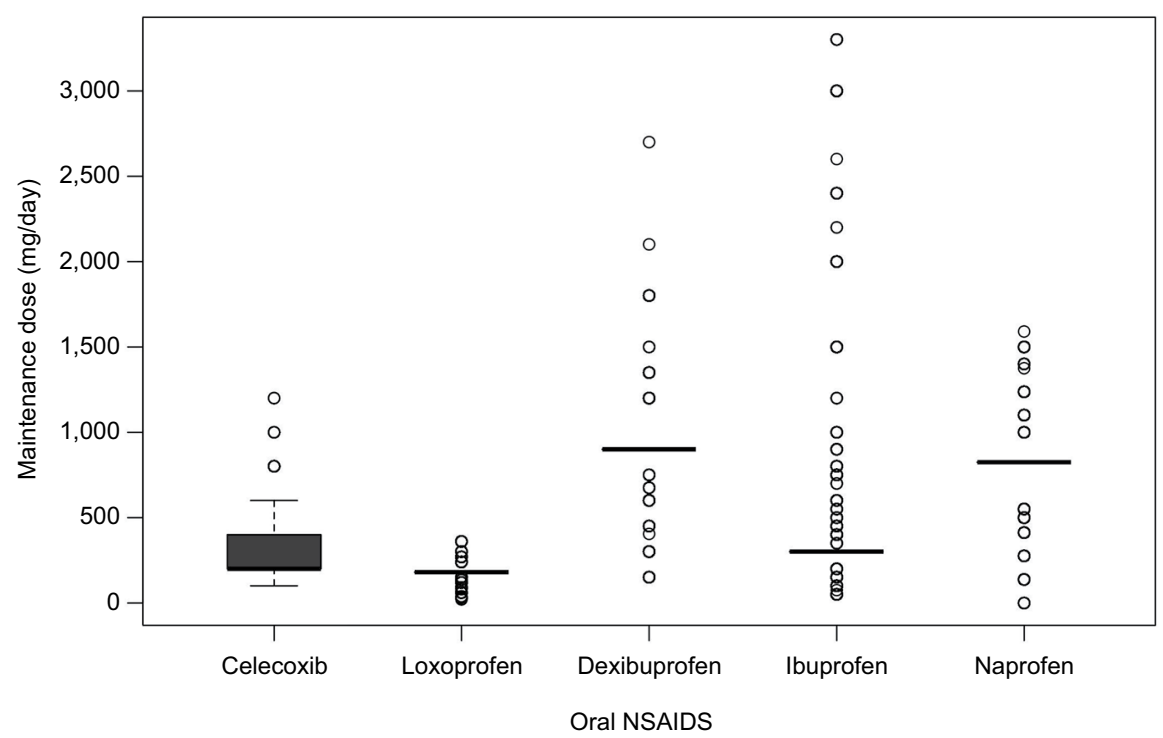

Figure I Overall maintenance daily dose of orally administered NSAIDs with therapy duration of 30 or more days. Abbreviation: NSAIDs, nonsteroidal anti-inflammatory drugs.

Table 5 PS-matched analysis for relative risks of GI, CV, and renal events associated with high ( $>400 \mathrm{mg} /$ day) and low-to-moderate ( $\leq 400 \mathrm{mg} /$ day) doses of celecoxib (treatment duration of 30 or more days)

\begin{tabular}{|c|c|c|c|c|}
\hline \multirow[t]{2}{*}{ Outcomes } & $\begin{array}{l}\text { High dose of } \\
\text { celecoxib }\end{array}$ & $\begin{array}{l}\text { Low to moderate } \\
\text { dose of celecoxib }\end{array}$ & \multicolumn{2}{|l|}{$\begin{array}{l}\text { High dose vs } \\
\text { low dose }\end{array}$} \\
\hline & \multicolumn{2}{|c|}{ Number of patients (percent) } & OR $(95 \% \mathrm{Cl})$ & $P$-value \\
\hline GI bleeding & $10(2.9)$ & $771(1.1)$ & $2.77(1.47-5.22)$ & 0.005 \\
\hline CV diseases & $162(46.3)$ & $27,472(37.4)$ & $1.44(1.17-1.78)$ & $<0.001$ \\
\hline Ischemic stroke & $95(27.1)$ & $14,477(19.7)$ & $1.52(1.20-1.92)$ & $<0.001$ \\
\hline Transient ischemic attack & $8(2.3)$ & $1,936(2.6)$ & $0.86(0.43-1.74)$ & 0.68 \\
\hline Unstable angina & $70(20.0)$ & $12,247(16.7)$ & $1.25(0.96-1.62)$ & 0.10 \\
\hline Acute myocardial infarction & $12(3.4)$ & $\mathrm{I}, 416(\mathrm{I} .9)$ & $1.80(1.01-3.22)$ & 0.04 \\
\hline Chronic ischemic heart disease & $26(7.4)$ & $3,663(5.0)$ & $1.53(1.02-2.28)$ & 0.04 \\
\hline Heart failure & $48(13.7)$ & $6,500(8.9)$ & $1.64(1.21-2.22)$ & 0.001 \\
\hline Coronary revascularization & $3(0.9)$ & $428(0.6)$ & NA & NA \\
\hline $\mathrm{PCl}$ & $3(0.9)$ & $409(0.6)$ & NA & NA \\
\hline CABG & $0(0)$ & $22(0.03)$ & NA & NA \\
\hline Renal events & $22(6.3)$ & $2,4 I I(3.3)$ & $1.97(1.28-3.05)$ & 0.002 \\
\hline
\end{tabular}

Notes: $P$-values were estimated by chi-squared test or Fisher's exact test. Statistically significant $P$-values are highlighted in bold.

Abbreviations: $\mathrm{CABG}$, coronary artery bypass graft; $\mathrm{Cl}$, confidence interval; $\mathrm{CV}$, cardiovascular; $\mathrm{Gl}$, gastrointestinal; $\mathrm{NA}$, not applicable; OR, odds ratio; $\mathrm{PCl}$, percutaneous coronary intervention; PS, propensity score.

mechanism of selective COX-2 inhibition. ${ }^{3}$ However, such a relationship was not observed in other strata of shorter treatment as well as when all strata were assessed collectively. The subgroup analysis, including only those patients without co-medication with PPIs or H2RAs for gastric protection, showed no evidence of improved GI consequences with celecoxib relative to traditional NSAIDs in all strata combined as well as separately in each stratum. With regards to CV and renal toxicities, a tendency toward greater risk of endpoint events was found with celecoxib vs traditional NSAIDs; OR values with statistical significance were generally higher with longer therapy, suggesting cumulative adverse effects of more prolonged exposure to celecoxib.

Celecoxib was one of the most frequently prescribed COX inhibitors, despite lingering safety concerns about a possible class effect similar to those with other COX-2 specific inhibitors; previous coxibs had been withdrawn from the market due to CV risks. ${ }^{5}$ Several studies have 
been conducted to evaluate differential risks associated with celecoxib vs traditional NSAIDs: some to clinically confirm the theoretical GI benefits of the selective inhibition of COX-2, ${ }^{10,12-16}$ and others to verify the controversial $\mathrm{CV}$ safety of the sole remaining coxib agent. ${ }^{7,17-19}$ Evidence is accumulating that celecoxib may be a better alternative to traditional NSAIDs in patients at risk of GI bleeding in that it theoretically retains equivalent analgesic and antiinflammatory efficacy, but with improved GI tolerability relative to traditional NSAIDs. ${ }^{12}$ However, in many studies, the co-prescribing of gastroprotective PPIs or H2RAs were permitted in both treatment arms, potentially functioning as a confounder affecting the GI bleeding rates.

In accordance with the FDA's mandate for reassurance on long-term CV safety of celecoxib, a postmarketing clinical trial titled the Prospective Randomized Evaluation of Celecoxib Integrated Safety vs Ibuprofen or Naproxen (PRECISION) was conducted. ${ }^{8}$ The trial findings revealed that celecoxib at moderate doses (up to $400 \mathrm{mg}$ /day) was noninferior to ibuprofen or to naproxen in terms of CV adverse outcomes. ${ }^{8}$ However, the PRECISION trial had a major flaw in its design: due to regulatory restrictions, celecoxib up-titration was not permitted particularly in $89.9 \%$ of study patients with osteoarthritis, while the doses of the comparator NSAIDs could have been escalated as needed for symptom management. Hence, the dose of celecoxib may not have been equipotent to that of comparator drugs, potentially confounding the results: administering a higher dose of the comparator NSAIDs was more effective in reducing pain, but perhaps put patients at an increased risk of developing adverse GI, $\mathrm{CV}$, and renal events as compared to celecoxib use with dose capping. Interestingly, the findings in the current study in general indicated an increased risk of $\mathrm{CV}$ consequences with celecoxib relative to traditional NSAIDs, which might have been influenced by higher-than-approved doses of celecoxib used in a considerable proportion of patients included herein. Despite the dosing guidelines recommendations with regard to dose escalation up to $200 \mathrm{mg}$ /day for osteoarthritis patients and up to $400 \mathrm{mg} /$ day for rheumatoid arthritis patients, it was noted that $24.3 \%$ of osteoarthritis-only patients on celecoxib received more than $200 \mathrm{mg} /$ day, and, more importantly, $4.7 \%$ of celecoxib-treated patients received more than $400 \mathrm{mg} /$ day in the current study.

The dose-dependent relationship between GI/CV/renal adverse consequences and celecoxib exposure was also confirmed in the additional analysis (Table 5). The findings from the PRECISION trial did not provide evidence on the safety of high-dose celecoxib because only moderate doses (up to $200 \mathrm{mg} /$ day for osteoarthritis patients and up to 400 $\mathrm{mg}$ /day for rheumatoid arthritis patients) were provided for study participants. Given that older patients requiring daily pain and inflammation management are at a greater risk of $\mathrm{CV}$ and renal toxicities owing to the chronic exposure to COX inhibitors, it is of significant importance that upward dosing titration for celecoxib should be strictly restricted by regulatory actions as in the US and Europe to moderate doses up to $200 \mathrm{mg} /$ day in most cases, but up to $400 \mathrm{mg} /$ day in rheumatoid arthritis patients. ${ }^{20}$ Older patients with arthritis suffering from mild-to-moderate pain may benefit from receiving a carefully designed combination regimen with dual oral analgesics (eg, celecoxib combined with acetaminophen), such that celecoxib doses can be reduced and that intermittent drug holidays can be implemented when appropriate.

Notably, it appeared that a sizable proportion of older patients with osteoarthritis or rheumatoid arthritis in the current study were receiving more than the maximum approved or recommended doses of celecoxib for their indications. Daily therapy with high-dose celecoxib in the long-term can render those patients with multiple comorbidities and risk factors along with already decreased renal function more vulnerable to its potential $\mathrm{CV}$ and renal toxicity. Although growing evidence showed that the safety profile of celecoxib at moderate doses is generally comparable to that of NSAIDs, ${ }^{8}$ more research designed to account for potential confounding due to concomitant use of gastroprotective drugs and to address potential limitations of controlled clinical trials is needed to ascertain whether celecoxib's GI benefits would outweigh its other possible risks with regard to $\mathrm{CV}$ and renal complications as compared to traditional NSAIDs.

This study has several limitations. First, any patients in long-standing remission who did not require medical attention in 2016 might not have been identified as rheumatoid arthritis patients. Second, information on relevant laboratory findings regarding metabolic functions and potential confounders, such as overall health status of study patients and severity of condition, was not available in the claims data. Third, any diagnostic codes or prescription data not documented correctly could have affected the study results. Fourth, it was assumed that the whole course of therapy was completed as prescribed, despite potential non-compliance in older patients. Lastly, $\mathrm{CV}$ outcomes that require longer follow-up were not captured due to the limited study period.

\section{Conclusion}

Celecoxib was associated with decreased risk of GI bleeding compared with traditional NSAIDs when treatment lasted for 
120 days and more, but such a relationship was not found in sub-group patients with no concomitant use of PPIs or H2RAs. CV and renal risks were higher with celecoxib than with traditional NSAIDs, and a dose-dependent risk relationship was suggested among celecoxib-treated patients.

\section{Acknowledgments}

This study was supported by a Basic Science Research Program through the National Research Foundation of Korea (NRF), funded by the Ministry of Science, Information and Communications Technology (ICT) \& Future Planning (No 2017R1C1B5015912). The national patient data for the analysis were provided by the Korean Health Insurance Review and Assessment Service (HIRA). The contents of this research do not represent the official views of the HIRA.

\section{Disclosure}

The author reports no conflicts of interest in this work.

\section{References}

1. Hochberg MC, Altman RD, April KT, et al. American College of Rheumatology 2012 recommendations for the use of nonpharmacologic and pharmacologic therapies in osteoarthritis of the hand, hip, and knee. Arthritis Care Res. 2012;64(4):465-474.

2. Zhang W, Doherty M, Arden N, et al. EULAR evidence based recommendations for the management of hip osteoarthritis: report of a task force of the EULAR Standing Committee for International Clinical Studies Including Therapeutics (ESCISIT)s. Ann Rheum Dis. 2005;64(5):669-681.

3. Whittle BJ. COX-1 and COX-2 products in the gut: therapeutic impact of COX-2 inhibitors. Gut. 2000;47(3):320-325.

4. Wolfe MM, Lichtenstein DR, Singh G. Gastrointestinal toxicity of nonsteroidal antiinflammatory drugs. $N$ Engl J Med. 1999;340(24): 1888-1899.

5. Zarghi A, Arfaei S. Selective COX-2 Inhibitors: A Review of Their Structure-Activity Relationships. Iran J Pharm Res. 2011;10(4):655-683.

6. Fitzgerald GA. Coxibs and cardiovascular disease. $N$ Engl J Med. 2004;351(17):1709-1711.
7. Solomon SD, Mcmurray JJ, Pfeffer MA, et al. Cardiovascular risk associated with celecoxib in a clinical trial for colorectal adenoma prevention. $N$ Engl J Med. 2005;352(11):1071-1080.

8. Nissen SE, Yeomans ND, Solomon DH, et al. Cardiovascular Safety of Celecoxib, Naproxen, or Ibuprofen for Arthritis. $N$ Engl J Med. 2016;375(26):2519-2529.

9. Mcgettigan P, Henry D. Cardiovascular risk with non-steroidal antiinflammatory drugs: systematic review of population-based controlled observational studies. PLoS Med. 2011;8(9):e1001098.

10. Chan FK, Hung LC, Suen BY, et al. Celecoxib versus diclofenac plus omeprazole in high-risk arthritis patients: results of a randomized double-blind trial. Gastroenterology. 2004;127(4):1038-1043.

11. Rahme E, Barkun AN, Toubouti Y, Scalera A, Rochon S, Lelorier J. Do proton-pump inhibitors confer additional gastrointestinal protection in patients given celecoxib? Arthritis Rheum. 2007;57(5):748-755.

12. Cryer B, Li C, Simon LS, Singh G, Stillman MJ, Berger MF. GI-REASONS: a novel 6-month, prospective, randomized, open-label, blinded endpoint (PROBE) trial. Am J Gastroenterol. 2013;108(3):392-400.

13. Silverstein FE, Faich G, Goldstein JL, et al. Gastrointestinal toxicity with celecoxib vs nonsteroidal anti-inflammatory drugs for osteoarthritis and rheumatoid arthritis: the CLASS study: A randomized controlled trial. Celecoxib Long-term Arthritis Safety Study. JAMA. 2000;284(10):1247-1255.

14. Chan FK, Wong VW, Suen BY, et al. Combination of a cyclo-oxygenase-2 inhibitor and a proton-pump inhibitor for prevention of recurrent ulcer bleeding in patients at very high risk: a double-blind, randomised trial. Lancet. 2007;369(9573):1621-1626.

15. Chan FK, Lanas A, Scheiman J, Berger MF, Nguyen H, Goldstein JL. Celecoxib versus omeprazole and diclofenac in patients with osteoarthritis and rheumatoid arthritis (CONDOR): a randomised trial. Lancet. 2010;376(9736):173-179.

16. Goldstein JL, Cryer B, Amer F, Hunt B. Celecoxib plus aspirin versus naproxen and lansoprazole plus aspirin: a randomized, double-blind, endoscopic trial. Clin Gastroenterol Hepatol. 2007;5(10):1167-1174.

17. Hirayama A, Tanahashi N, Daida H, et al. Assessing the cardiovascular risk between celecoxib and nonselective nonsteroidal antiinflammatory drugs in patients with rheumatoid arthritis and osteoarthritis. Circ J. 2014;78(1):194-205.

18. White WB, West CR, Borer JS, et al. Risk of cardiovascular events in patients receiving celecoxib: a meta-analysis of randomized clinical trials. Am J Cardiol. 2007;99(1):91-98.

19. Caldwell B, Aldington S, Weatherall M, Shirtcliffe P, Beasley R. Risk of cardiovascular events and celecoxib: a systematic review and metaanalysis. J R Soc Med. 2006;99(3):132-140.

20. Slomski A. Celecoxib Similar to 2 NSAIDs for Cardiovascular Safety. JAMA. 2016;316(24):2589. 


\section{Supplementary material}

Table SI Breakdown of all nonsteroidal anti-inflammatory drugs (NSAIDs) included in this study

\begin{tabular}{|c|c|c|c|}
\hline Order & Substance name & Number of prescriptions & $\%$ of prescription volume \\
\hline I & Loxoprofen & $2,156,592$ & 31.6 \\
\hline 2 & Celecoxib & 847,907 & 12.4 \\
\hline 3 & Dexibuprofen & 844,104 & 12.4 \\
\hline 4 & Talniflumate & 603,058 & 8.8 \\
\hline 5 & Meloxicam & 538,260 & 7.9 \\
\hline 6 & Diclofenac & 493,466 & 7.2 \\
\hline 7 & Mefenamic acid & 262,021 & 3.8 \\
\hline 8 & Ibuprofen & 248,529 & 3.6 \\
\hline 9 & Naproxen & 221,955 & 3.3 \\
\hline 10 & Zaltoprofen & 221,894 & 3.3 \\
\hline II & Piroxicam & $|43,48|$ & 2.1 \\
\hline 12 & Nabumetone & 57,212 & 0.8 \\
\hline 13 & Ketorolac & 50,366 & 0.7 \\
\hline 14 & Ketoprofen & 34,272 & 0.5 \\
\hline 15 & Etodolac & 33,145 & 0.5 \\
\hline 16 & Nimesulide & 23,216 & 0.3 \\
\hline 17 & Proglumetacin & 19,749 & 0.3 \\
\hline 18 & Pranoprofen & 13,778 & 0.2 \\
\hline 19 & Sulindac & 6,414 & 0.1 \\
\hline 20 & Flurbiprofen & 1,959 & 0.0 \\
\hline 21 & Tiaprofenic acid & 1,308 & 0.0 \\
\hline 22 & Imidazole salicylate & 1,230 & 0.0 \\
\hline 23 & Cinnoxicam & 457 & 0.0 \\
\hline 24 & Fenoprofen & 126 & 0.0 \\
\hline 25 & Salsalate & 83 & 0.0 \\
\hline 26 & Oxaprozin & 3 & 0.0 \\
\hline Total & & $6,824,585$ & 100 \\
\hline
\end{tabular}

Journal of Pain Research

\section{Publish your work in this journal}

The Journal of Pain Research is an international, peer reviewed, open access, online journal that welcomes laboratory and clinical findings in the fields of pain research and the prevention and management of pain. Original research, reviews, symposium reports, hypothesis formation and commentaries are all considered for publication.

\section{Dovepress}

The manuscript management system is completely online and includes a very quick and fair peer-review system, which is all easy to use. Visit http://www.dovepress.com/testimonials.php to read real quotes from published authors. 